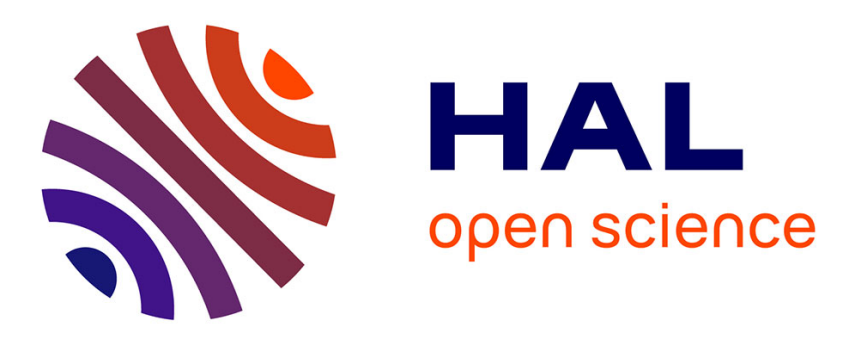

\title{
Breaking of rotational symmetry in a swirling jet experiment
}

\author{
Thomas Loiseleux, Jean-Marc Chomaz
}

\section{To cite this version:}

Thomas Loiseleux, Jean-Marc Chomaz. Breaking of rotational symmetry in a swirling jet experiment. Physics of Fluids, 2003, 15 (2), pp.511-523. 10.1063/1.1533068 . hal-01024930

\section{HAL Id: hal-01024930 \\ https://hal-polytechnique.archives-ouvertes.fr/hal-01024930}

Submitted on 27 Aug 2014

HAL is a multi-disciplinary open access archive for the deposit and dissemination of scientific research documents, whether they are published or not. The documents may come from teaching and research institutions in France or abroad, or from public or private research centers.
L'archive ouverte pluridisciplinaire HAL, est destinée au dépôt et à la diffusion de documents scientifiques de niveau recherche, publiés ou non, émanant des établissements d'enseignement et de recherche français ou étrangers, des laboratoires publics ou privés. 


\section{AIP $\mid$ Physics of Fluids}

\section{Breaking of rotational symmetry in a swirling jet experiment}

Thomas Loiseleux and Jean-Marc Chomaz

Citation: Physics of Fluids (1994-present) 15, 511 (2003); doi: 10.1063/1.1533068

View online: http://dx.doi.org/10.1063/1.1533068

View Table of Contents: http://scitation.aip.org/content/aip/journal/pof2/15/2?ver=pdfcov

Published by the AIP Publishing

\section{Articles you may be interested in}

Stability and temporal evolution of a swirling jet with centrifugally unstable azimuthal velocity

Phys. Fluids 14, 4081 (2002); 10.1063/1.1509755

Temporal instability of swirling gas jets injected in liquids

Phys. Fluids 13, 2845 (2001); 10.1063/1.1396670

Viscosity influence on the stability of a swirling jet with nonrotating core

Phys. Fluids 12, 1607 (2000); 10.1063/1.870409

Three-dimensional instability of anticyclonic swirling flow in rotating fluid: Laboratory experiments and related theoretical predictions

Phys. Fluids 10, 3194 (1998); 10.1063/1.869846

The effect of swirl on jets and wakes: Linear instability of the Rankine vortex with axial flow Phys. Fluids 10, 1120 (1998); 10.1063/1.869637

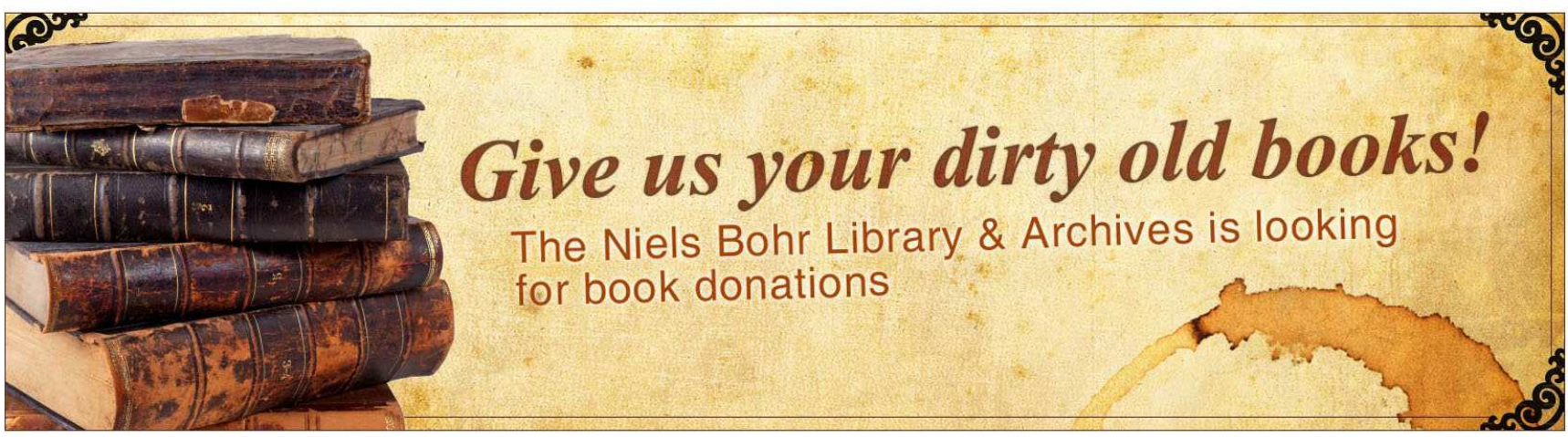




\title{
Breaking of rotational symmetry in a swirling jet experiment
}

\author{
Thomas Loiseleux ${ }^{\mathrm{a})}$ and Jean-Marc Chomaz \\ Laboratoire d'Hydrodynamique (LadHyX), CNRS-Ecole Polytechnique, 91128 Palaiseau Cedex, France
}

(Received 5 August 2002; accepted 6 November 2002; published 8 January 2003)

\begin{abstract}
In this experimental investigation, the dynamics of symmetry-breaking instabilities in swirling jets is analyzed for swirl parameters $S$ in the pre-breakdown range $0 \leqslant S \leqslant S_{c}$, where $S_{c}=1.3$ is the critical swirl value for the appearance of vortex breakdown determined by Billant, Chomaz, and Huerre [J. Fluid Mech. 376, 183 (1998)]. As $S$ is increased, three distinct dynamical regimes have been identified in the streamwise region extending to the end of the potential core. In the low swirl range $S<0.6$, the evolution is governed by the same instability mechanisms as in a nonswirling jet. The shear in axial direction generates axisymmetric vortex rings at a Strouhal number independent of the swirl $S$. As $S$ increases, the amplitude of the axisymmetric mode decreases in magnitude. Concurrently, co-rotating streamwise vortices form in the braids connecting the rings due to a secondary instability mechanism. The advection by the mean rotation of these secondary structures generates an azimuthal wave propagating cyclonically when compared to the imposed rotation, at a phase velocity proportional to swirl. When swirl reaches the transitional swirl level $S \sim 0.6$, no azimuthal or standing wave is observed, and the swirling jet is completely dominated by the development of the axisymmetric mode into ring vortices. In the intermediate swirl range $0.6<S$ $\leqslant 1$, vortex rings form concurrently with several interacting helical cyclonic waves of azimuthal wave number 2 . The mean phase velocity of the resulting propagating wave increases at a constant rate with swirl, and much more rapidly than in the low swirl regime. In this swirl range, azimuthal and axisymmetric deformations are of comparable high levels. In the high swirl range $1<S$ $<1.3$, another step toward complexity is reached, and there is a strong interaction between the azimuthal waves and the ringlike structures. The most striking feature of this flow regime is the emergence of a bending mode $m=1$ propagating with a high negative phase velocity. (C) 2003 American Institute of Physics. [DOI: 10.1063/1.1533068]
\end{abstract}

\section{INTRODUCTION}

The objective of the present experimental study is to describe and analyze the dynamics of instability waves in a hydraulic jet as swirl is gradually increased up to the critical value for the onset of vortex breakdown.

In nonswirling jets, the development of instability waves is known to account for the flow dynamics: in a large Reynolds number range, jets are dominated by ringlike vortical structures induced by the primary Kelvin-Helmholtz shear instability. A secondary three-dimensional instability breaks the rotational symmetry of the vortex rings and leads to the formation of streamwise counter-rotating vortex pairs in the braids. The mechanism responsible for the generation of these secondary structures has been shown by $\mathrm{Neu}^{1}$ and Lin and $\operatorname{Corcos}^{2}$ to involve an instability of the vortex sheet in the braid region, resulting in the collapse of the braid vorticity into streamwise vortices. These vortices appear in crosssectional images as mushroom-shaped structures surrounding the jet, or as azimuthal deformations at the early stage of their development.

The effect of rotation on the development of the primary

\footnotetext{
a) Author to whom correspondence should be addressed. Present address: Unité de Mécanique, Ecole Nationale Supérieure de Techniques Avancées (ENSTA), 32 boulevard Victor, 75739 Paris Cedex 15, France. Electronic mail: thomas.loiseleux@ensta.fr
}

instability has been well documented by numerous theoretical investigations that are summarized below. Interest in columnar vortex dynamics has first led to the establishment of criteria for instability. The studies of Rayleigh ${ }^{3}$ and Synge ${ }^{4}$ have concluded that a necessary and sufficient condition for centrifugal instability of the axisymmetric mode $m=0$ is given by

$$
\frac{d}{d r}(r W)^{2}<0
$$

where $r$ is the radial distance to the vortex axis and $W(r)$ the azimuthal velocity distribution in the radial direction. In other words, this criterion means that a vortex is unstable with respect to axisymmetric perturbations if and only if the circulation decreases as the radial distance increases. Howard and Gupta ${ }^{5}$ have generalized this criterion to columnar vortices with axial flow $U(r)$ and obtained the following necessary condition for the instability of the axisymmetric mode $m=0$ :

$$
\frac{d}{d r}(r W)^{2}<\frac{1}{4} r^{3}\left(\frac{d U}{d r}\right)^{2}
$$

Leibovich and Stewartson ${ }^{6}$ later proved that a sufficient condition for instability with respect to helical perturbations $m$ $\neq 0$ is given by 


$$
\frac{d}{d r}\left(\frac{W}{r}\right)\left[\frac{d}{d r}\left(\frac{W}{r}\right) \frac{d}{d r}(W r)+\left(\frac{d U}{d r}\right)^{2}\right]<0 .
$$

A different theoretical approach has consisted in directly determining the eigenmodes of a columnar vortex of azimuthal velocity $W(r)$ with axial flow $U(r)$. Lessen, Singh, and Paillet ${ }^{7}$ have studied the temporal inviscid linear stability of the "Batchelor vortex," with respect to helical perturbations of the form $e^{i(k x+m \theta-\omega t)}$, as the swirl parameter $S$ that compares the relative intensities of the azimuthal and axial velocities of the vortex core is gradually increased. The main conclusion of their study lies in the distinction between the behavior of the positive and negative helical modes $m$. Without swirl, the only unstable modes are $|m|=1$. When swirl is increased, the $m=+1$ mode is rapidly damped, whereas its negative counterpart $m=-1$ is amplified, and all the negative helical modes $m \leqslant-2$ are destabilized. Maximum growth rates $\omega_{i}^{\max }(m ; S)$ increase continuously with the azimuthal wave number modulus $|m|$ and maximum amplification occurs for $S \sim 0.85$. For swirl levels higher than 0.85 , the instability modes experience gradually decreasing growth rates and they become all damped for $S \geqslant 1.5$. Analogous viscous studies by Lessen and Paillet, ${ }^{8}$ Khorrami, ${ }^{9}$ and Mayer and Powell ${ }^{10}$ have revealed the existence of distinct families of purely viscous modes, with growth rates much weaker than their inviscid counterparts. It is worth noting that this vortex model does not exhibit any instability of the axisymmetric mode $m=0$, even without swirl. This feature suggests that the Batchelor vortex is in a sense inadequate: it does not properly reproduce the strong shear in axial direction observed in jet or swirling jet experiments (Panda and McLaughlin, ${ }^{11}$ Billant, Chomaz, and Huerre ${ }^{12}$ ).

Recently, Loiseleux, Chomaz, and Huerre ${ }^{13}$ analyzed the inviscid linear instability properties of a Rankine vortex with axial plug flow velocity. In contrast with the previous model, the axisymmetric mode $m=0$ is unstable. The effect of increasing swirl on $m=0$ is to lower the amplification rate $\omega_{i}(0 ; S)$ for all axial wave numbers $k$. Without swirl, all helical wave numbers $|m| \neq 0$ are also unstable. The introduction of swirl breaks the rotational symmetry and leads to distinct temporal instability properties for helical modes $+m$ and $-m$ : swirl leads to larger growth rates for negative helical modes than for their positive counterparts. For low axial wave numbers $k$, small amounts of swirl lead to the widespread occurrence of direct resonances between the unstable Kelvin-Helmholtz wave induced by the shear and the countable infinity of neutrally stable inertial waves generated by the solid body rotation. This feature results in the appearance of infinitely many bubbles of instability separated by neutrally stable wave number bands. The growth rate envelope decreases continuously with increasing swirl and neutrally stable bands vanish beyond a critical swirl value. For high axial wave numbers $k$, low swirl levels are destabilizing and large ones are stabilizing for negative helical modes $(m$ $<0)$. For positive helical modes $(m>0)$, increasing swirl is always stabilizing. At low axial wave numbers $k, m=0$ is the most unstable mode and $|m|=\infty$ the least unstable, whereas at high wave numbers, the growth rate $\omega_{i}(m ; S)$ decreases continuously with $m: m=-\infty$ is then the most unstable mode. Note that high wave numbers always remain unstable due to the infinitely thin shear layer thickness of the axial velocity profile. This feature is a strong limitation of this idealized model.

Both, the "Batchelor vortex" and the "Rankine vortex" models are centrifugally stable, and they fail in reproducing the rapid decrease of circulation away from the vortex axis experimentally observed in swirling jets. Martin and Meiburg $^{14}$ have determined the inviscid linear instability properties of a family of swirling jet shear layers consisting of a straight vortex filament of circulation $\Gamma_{c}$ surrounded by a cylindrical vortex sheet of strength $\Gamma_{c}-\Gamma_{\infty}$, where $\Gamma_{\infty}$ is the external circulation. A plug flow axial jet velocity profile is added to this purely swirling cylindrical vortex sheet. For centrifugally stable flows $\left(\Gamma_{c}-\Gamma_{\infty}<0\right)$, the instability is of Kelvin-Helmholtz type, feeding on both azimuthal and axial vorticity, and negative helical waves $(m<0)$ are the most unstable. When the flow is centrifugally unstable $\left(\Gamma_{c}-\Gamma_{\infty}\right.$ $>0$ ), the most unstable modes consist of positive helical modes $(m>0)$. A straightforward application of the explicit dispersion relation given by Martin and Meiburg reveals that centrifugally stable situations lower the amplification rate of all helical and axisymmetric modes at all axial wave numbers, and that centrifugally unstable situations amplifies all $m$ at all $k$.

The first experimental observations of instability modes in a vortex with axial flow have been conducted by Singh and Uberoi. ${ }^{15}$ These authors studied the tip vortex of a laminar flow wing and found the existence of an axisymmetric mode $m=0$ and of a bending mode $|m|=1$ of instability. A more recent study by Panda and McLaughlin ${ }^{11}$ was aimed at understanding the development of Kelvin-Helmholtz instability waves and of the resulting organized structures in a free swirling air jet for Reynolds numbers in the range $20000 \leqslant \operatorname{Re} \leqslant 60000$. High swirl levels preceding breakdown were investigated. According to their observations, a further increase in swirl produced a highly turbulent jet due to the appearance of vortex breakdown. In these experiments, the azimuthal velocity component behaved as in a solid body rotation near the jet centerline. Away from the centerline, the azimuthal velocity radial distribution was similar to a potential vortex. An acoustic forcing system was arranged circumferentially around the jet exit, consisting of four large acoustic speakers. Various phase combinations between speakers were possible and different instability modes could be excited. In the absence of forcing, velocity spectra in the shear layer at different streamwise stations did not show significant streamwise growth, in contrast with the observations made in a nonswirling jet. However, acoustic excitation successfully produced regular organized structures of both axisymmetric $(m=0)$ and helical $(m=+1$ and $m=-1)$ modes. It was concluded that swirl had a softening effect on the amplification of unstable modes.

The lack of experimental results has not so far led to many numerical studies. However, the investigations of Martin and Meiburg ${ }^{16}$ give interesting insights on the effect of swirl on jets. The model is the same as in Martin and Meiburg: ${ }^{14}$ as previously stated, it consists of a straight vortex filament of circulation $\Gamma_{c}$ surrounded by a cylindrical 
vortex sheet of strength $\Gamma_{\infty}$, superimposed on a plug flow axial velocity profile. The external circulation $\Gamma_{\infty}$ is chosen to be zero. This simple velocity distribution enables to study the nonlinear interaction and competition of the various instability mechanisms involved, namely the shear in axial direction, the shear in azimuthal direction, and the centrifugal instability, by means of a fully nonlinear numerical scheme based on the vortex filament technique. Without axial velocity component, Caflisch, Li, and Shelley ${ }^{17}$ have observed the formation of pairs of counter-rotating vortex rings of equal strength, characteristic of the centrifugal instability. With large swirl, the jet evolves similarly when subjected to axisymmetric forcing, but the Kelvin-Helmholtz instability of the axial flow amplifies one vortex ring at the expense of the other. An additional azimuthal perturbation leads to the formation of concentrated streamwise vortices as a result of the Kelvin-Helmholtz instability feeding on the streamwise vorticity. In contrast to nonswirling jets, the streamwise vortices are all of the same sign. The ratio between the perturbation amplitudes in the azimuthal and axial directions plays an important role in the subsequent swirling jet development. When the ratio is small, the centrifugal instability induces the formation of counter-rotating vortex rings, before concentrated streamwise vortices can emerge in the braid region. For higher amplitude ratios, streamwise vortices grow rapidly between the like-signed Kelvin-Helmholtz vortex rings and counter-rotating vortex rings are inhibited. For even higher ratios, co-rotating streamwise vortices grow rapidly and no vortex rings develop in the flow.

All the above considerations, whether theoretical, numerical or experimental, pertain to swirling jets below the critical swirl value $S_{c}=1.3$ signaling the onset of vortex breakdown, as determined by Billant, Chomaz, and Huerre. ${ }^{12}$ The present study is precisely restricted to this pre-vortex breakdown range $0 \leqslant S \leqslant S_{c}$. It should be viewed as a complement to the investigation in Ref. 12 which was, for the most part, devoted to the description of the various breakdown states observed above $S_{c}=1.3$. Note that according to Ref. 12, $S_{c}$ is independent of the Reynolds number in the range $300 \leqslant \operatorname{Re} \leqslant 1200$. The experimental set-up and procedure are outlined in Sec. II. Quantitative measurements presented in Sec. III lead to distinguish three different flow regimes as swirl $S$ is increased from zero to the critical value $S_{c}$. Section IV is devoted to a qualitative description of the different flow regimes previously identified. The main conclusions are summarized in Sec. V.

\section{EXPERIMENTAL SETUP AND PROCEDURE}

The experimental setup consists of a vertical water jet discharging into a large water tank as sketched in Fig. 1. The jet axial velocity is generated by the hydrostatic pressure difference between two constant head reservoirs. A swirl velocity component is imparted to the jet by means of a motor and two concentric cylinders in the following way (see Fig. 1 ): the outer cylinder is fixed and the inner cylinder is set into rotational motion by a motor, and connected to the outer cylinder by a bearing track. The rotation rate of the motor is maintained constant by means of a servo-control. An over-

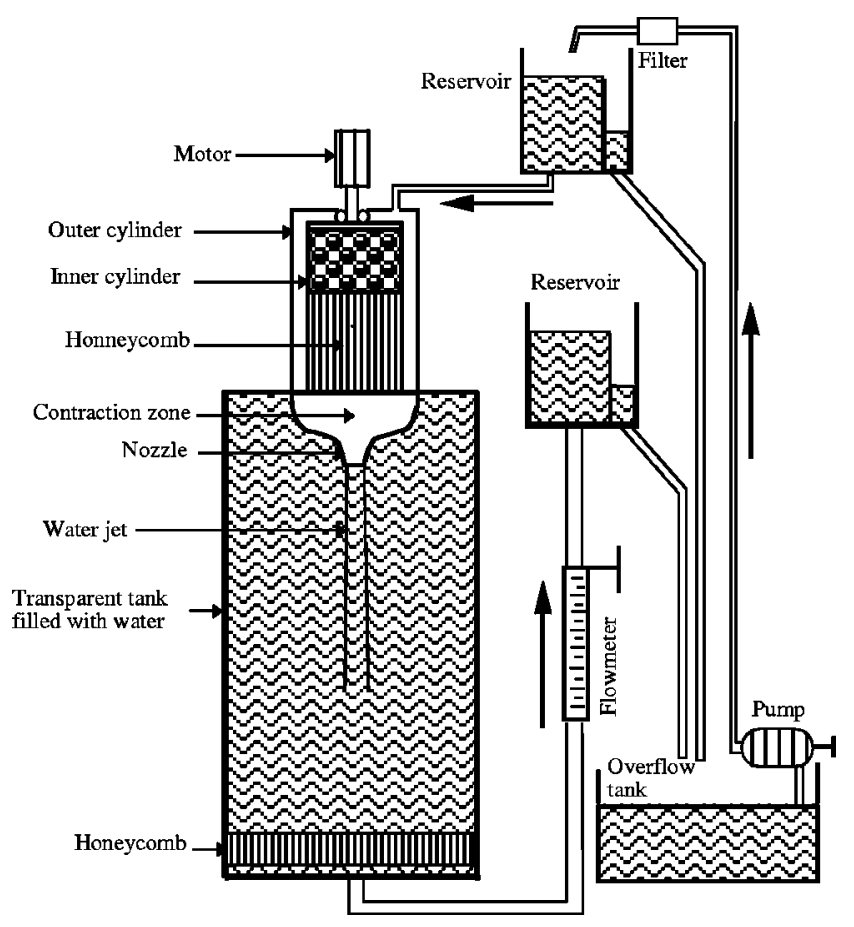

FIG. 1. Sketch of experimental apparatus.

flow tank and a pump close the water circuit. The flow rate is controlled by a valve and monitored with a flowmeter located on the exit pipe at the bottom of the tank. A filter on the exit pipe of the pump cleans the water from any impurities. The liquid is supplied from the upper reservoir to the outer cylinder through four pipes symmetrically placed on its top surface. Water is then fed to the inner cylinder through a network of holes regularly arranged on its upper part. The flow is kept laminar by inserting an $18 \mathrm{~cm}$ long honeycomb into the inner cylinder. The jet ends into a smooth converging nozzle attached to the outer cylinder and mounted on top of a large transparent tank of $120 \times 40 \times 40 \mathrm{~cm}^{3}$ square cross section (Fig. 1). The converging nozzle is designed according to the optimum method $\left(\right.$ Mikhail ${ }^{18}$ ) in order to avoid flow separation. The exit diameter of the contraction zone is $D$ $=2 R=40 \mathrm{~mm}$, with an area contraction ratio of $21.4: 1$ and a length-to-width ratio of 1.05 . It should be emphasized that the relative large size of the test tank minimizes the effect of confinement: measured recirculation currents were found by Billant, Chomaz, and Huerre ${ }^{12}$ to be negligible. To further ensure quiescent flow conditions, a honeycomb is placed at the bottom of the tank, upstream of the exit pipe (Fig. 1), in order to avoid any whirlpool to occur. Regular tap water is used but it is stocked in the same room several days ahead of time in order to eliminate air bubbles and to reach ambient temperature.

Throughout the study, a cylindrical coordinate system is used whereby $r, x$, and $\theta$ respectively refer to the radial, streamwise and azimuthal directions. Associated velocity components are denoted $U_{r}(r, x), U_{x}(r, x)$, and $U_{\theta}(r, x)$. As in Billant et al., ${ }^{12}$ the flow is controlled by two nondimensional parameters, the swirl and the Reynolds number. The swirl $S$ compares the relative intensities of the solid body rotation and the axial velocity. It is defined as 


$$
S=\frac{2 U_{\theta}\left(R / 2, x_{0}\right)}{U_{x}\left(0, x_{0}\right)}
$$

where the reference streamwise station $x_{0}=26 \mathrm{~mm}$ is the shortest axial distance from the nozzle exit at which measurements are possible. This definition is motivated by the fact that the angular velocity of the vortex core is approximately equal to $\Omega=U_{\theta}\left(R / 2, x_{0}\right) /(R / 2)$ so that effectively

$$
S \sim \frac{\Omega R}{U_{x}\left(0, x_{0}\right)} .
$$

The Reynolds number is defined as

$$
\operatorname{Re}=\frac{U_{x}^{S=0}\left(0, x_{0}\right) D}{\nu},
$$

where $U_{x}^{S=0}(r, x)$ denotes the axial velocity distribution in the absence of swirl and $\nu$ the kinematic viscosity. Two Reynolds numbers have been investigated: $\operatorname{Re}=740$ and $\mathrm{Re}$ $=1490$.

The axial and azimuthal velocity distributions $U_{x}(r, x)$ and $U_{\theta}(r, x)$ appearing in definitions (4) and (6) have been measured by laser Doppler anemometry. Details of the technique and typical velocity profiles for different swirl and Reynolds numbers may be found in Billant et al. ${ }^{12}$ Velocity profiles evolve smoothly when the control parameters are varied as shown in their Figs. 4 and 5.

In most cases, it has been found that very accurate values of the swirl parameter $S$ may be obtained without measuring the axial and azimuthal velocities at each Reynolds number and swirl as implied by definition (4). The procedure effectively used consists in exploiting the swirl law $S(\omega)$ $=a(1-\exp b \omega)\left(\right.$ determined by Billant et al. $\left.{ }^{12}\right)$ between $S$ and the motor angular velocity $\omega$, where the parameters $a$ and $b$ are experimentally determined constants. In contrast with Billant et al. ${ }^{12}$ the constants $a$ and $b$ are in the present case fitted by relying on the inviscid analytical model of Batchelor ${ }^{19}$ for the effect of a decreasing cross-section on a tube of fluid with uniform angular and axial velocity far upstream. The model provides analytical expressions for the velocities $U_{\theta}\left(R / 2, x_{0}\right)$ and $U_{x}\left(0, x_{0}\right)$ appearing in Eq. (4) as a function of the motor velocity $\omega$ and flow rate. It is found that the predicted values of $S$ are extremely close to the directly measured counterparts for low $S$. Furthermore, it is experimentally known (Billant et al. ${ }^{12}$ ) that breakdown always takes place at $S=1.3$, independently of the Reynolds number. The analytical predictions at low $S$, in conjunction with the breakdown point at $S=1.3$ are then invoked to determine the fitting constants $a$ and $b$ in the swirl law $S(\omega)$. Note that this procedure does not require detailed velocity measurements at each Reynolds number.

The homogeneity of water temperature in the whole circuit was carefully monitored: maximum temperature differences were kept below $0.04^{\circ} \mathrm{C}$, in order to avoid any buoyancy effects. Very low temperature gradients are known to drastically affect the behavior of the jet when it becomes lighter than the surrounding water (Billant et al. ${ }^{12}$ ). Let $R i$ $=\left(\rho_{0}-\rho_{\infty} / \rho_{\infty}\right)\left[g D / U_{x}^{S=0}\left(0, x_{0}\right)^{2}\right]$ denote the Richardson number based on the relative density difference $\left(\rho_{0}\right.$ $\left.-\rho_{\infty}\right) / \rho_{\infty}$ between the jet and the ambient medium. For values as low as $R i=-6.45 \times 10^{-3}$, corresponding to a jet velocity $U_{x}^{S=0}\left(0, x_{0}\right)=3.72 \mathrm{~cm} / \mathrm{s}$, and temperatures $T_{0}=22.1^{\circ} \mathrm{C}, T_{\infty}$ $=22.0^{\circ} \mathrm{C}$, the jet loses its axisymmetry at about three diameters downstream of the nozzle exit. The helical mode $m$ $=1$ is observed to become dominant instead of the axisymmetric mode $m=0$ and the three-dimensional secondary instability is found to be inhibited. This feature has not been systematically investigated in the context of the present study.

The flow is visualized by the laser induced fluorescence technique (LIF). Fluorescein dye is introduced in each of the four exit pipes of the upper constant head reservoir (Fig. 1). The light source consists of a $1 \mathrm{~W}$ argon laser. The light sheet produced by a cylindrical lens is shined in a horizontal plane perpendicular to the axis of the main jet. A black and white $740 \times 480$ pixels CCD camera views the light sheet from below at an angle of about $30^{\circ}$. In order to compensate for parallax effects, the center $\mathrm{C}$ and observed aspect ratio of the nozzle exit section are determined by image processing with the NIH Image software. ${ }^{20}$ The camera and the laser are mounted on a vertical traversing mechanism, thereby allowing visualizations at different streamwise distances $X=x / D$ from the nozzle exit.

The development of the streamwise and azimuthal deformations is documented by following the temporal evolution of the jet radius distribution $r(t, \alpha ; X)$ with azimuthal angle $\alpha$, at different streamwise stations $X$. It should be pointed out that the visualization technique used in this study is not a simple pathline tracking since the dyed passive tracer is correlated to the vorticity. Indeed, at the nozzle exit, the interface between dyed and clear water marks a vorticity surface. If viscosity is neglected, this surface deforms as a material surface according to Helmholtz theorem. As a consequence, the dyed interface visualizes the vorticity, its evolution and its roll-up. The azimuthal distributions $r(t, \alpha ; X)$ are obtained from cross-sectional images such as those displayed in Fig. 2. The radius is defined as the distance between the center $\mathrm{C}$ and the jet interface delimited by the fluorescein-saturated region (clear area of Fig. 2). The interface location is precisely determined as the point of maximum gradient in gray

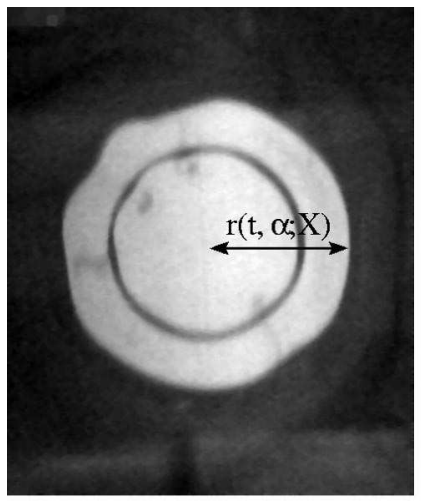

(a)

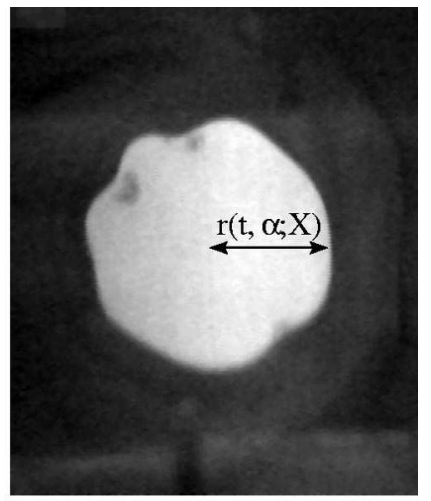

(b)
FIG. 2. LIF cross-sectional image of the jet at $X=3$ in the unforced case in the ring (a) and in the braid (b), for $\mathrm{Re}=1490$. 


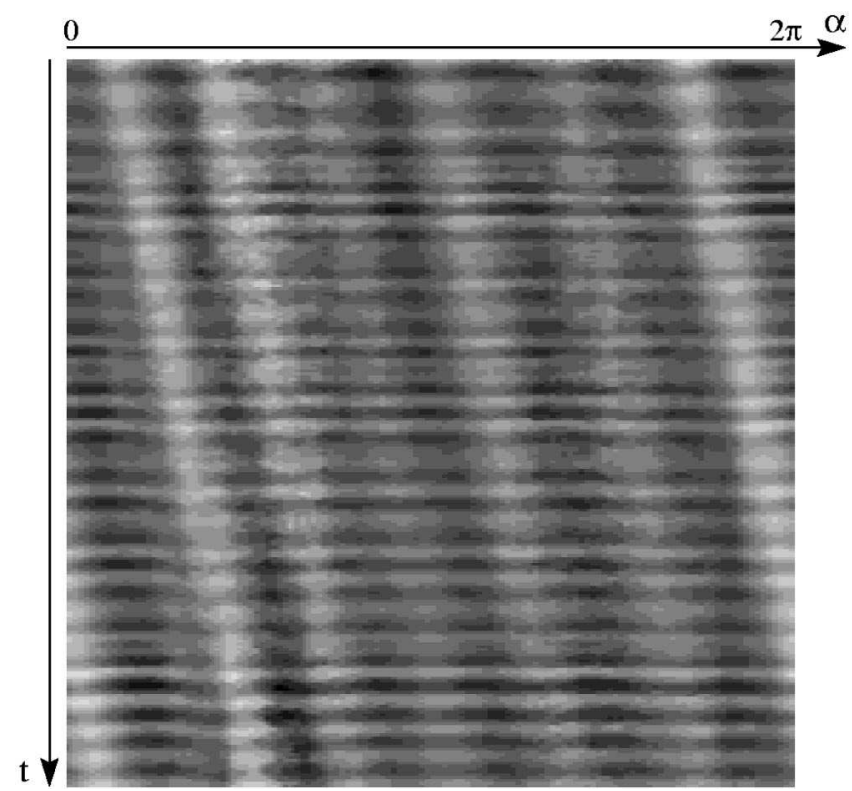

FIG. 3. Spatio-temporal $\alpha-t$ diagram for a swirling jet at $X=1, \operatorname{Re}$ $=1490$, and $S=0.38$. The sampling rate is $\Delta t=0.16 \mathrm{~s}$. The total recording time is $T=40.8 \mathrm{~s}$.

level intensity. Typical spatio-temporal $\alpha-t$ diagrams are then constructed as in Fig. 3, where time is increasing downward and the horizontal axis is the azimuthal angle $\alpha$. The angle $\alpha$ is taken to be positive in the cyclonic direction with respect to the jet rotation. Gray levels correspond to different radii, the lighter shades being associated with smaller radii. A typical sampling interval is $\Delta t=0.16 \mathrm{~s}$. Two sets of patterns are clearly identifiable: axisymmetric deformations appear as horizontal stripes, while inclined stripes represent travelling azimuthal deformations. The dominant azimuthal wave numbers and associated phase velocities are easily obtained by performing two-dimensional fast Fourier transforms of such diagrams. We should here emphasize that, in stability theory, the sign of $m$ refers to the winding of the three-dimensional helix and, the sign of the azimuthal phase velocity is defined by $\omega / m$ where both $\omega$ and $m$ may be positive or negative. In the present study, we do not have access to the axial coherence of the instability mode and, consequently, the azimuthal wave number $m$ remains unsigned. The flow visualization gives the time evolution of the mode in a single transversal plane and we directly measure the azimuthal phase velocity which is positive if the structure rotates cyclonically with respect to the base flow and negative when it rotates anticyclonically. For the axisymmetric mode $m=0$, the associated passage frequency $f_{l}$ of the ring vortices is determined by counting the number of horizontal stripes. However, when roll-up is too intense, this method is no longer suitable, and a more reliable way consists in following the temporal evolution of the jet diameter at different streamwise stations. A new spatio-temporal diagram is then constructed as in Fig. 4. Such a representation is generated by recording the time variations of a fixed radial line going through the jet center $C$ at a $0.04 \mathrm{~s}$ sampling rate. Note the pairing event over the first few cycles.

The $\alpha-t$ spatio-temporal diagram in Fig. 3, may be ex-

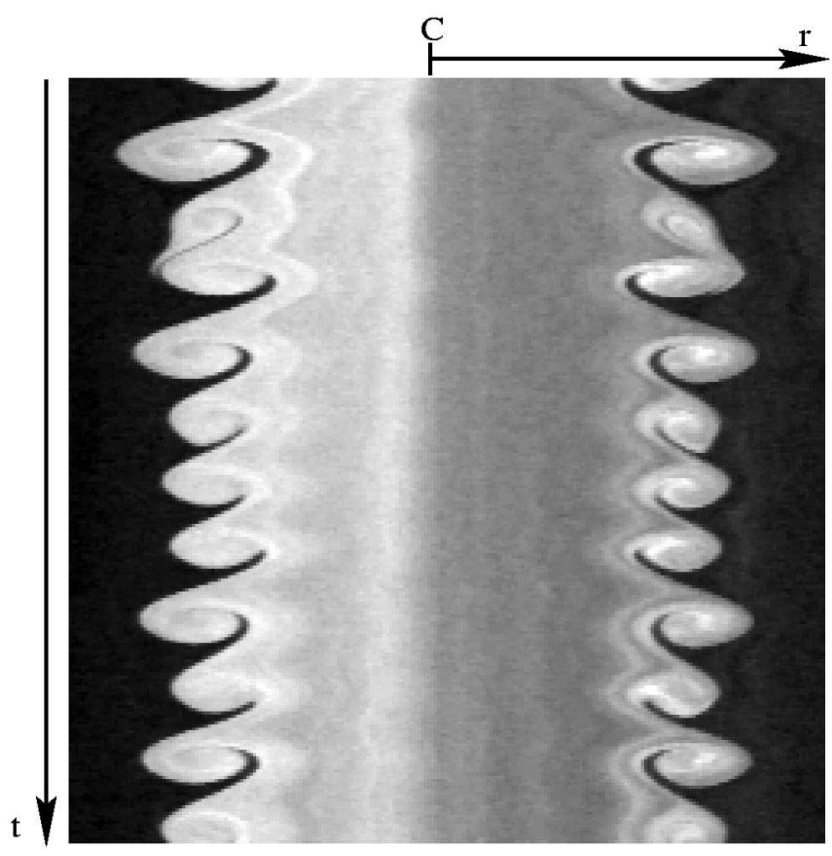

FIG. 4. Temporal evolution of jet diameter at $X=3$ and $\mathrm{Re}=1490$. The sampling rate is $\Delta t=0.08 \mathrm{~s}$. The total recording time is $T=19 \mathrm{~s}$.

ploited to yield detailed quantitative measures of the perturbation levels. The amplitude of the axisymmetric mode is determined from the standard deviation formula

$$
A(X ; S)=\sqrt{3} \frac{\overline{\sqrt{\left\langle r(t, \alpha ; X)^{2}\right\rangle-\langle r(t, \alpha ; X)\rangle^{2}}}}{D / 2}
$$

and the amplitude of the azimuthal deformations is correspondingly given by

$$
a(X ; S)=\sqrt{3} \frac{\left\langle\sqrt{\left.\overline{r(t, \alpha ; X)^{2}}-\overline{r(t, \alpha ; X)^{2}}\right\rangle}\right.}{D / 2},
$$

where $\langle\cdot\rangle$ denotes the time average, and a bar the azimuthal average over $0 \leqslant \alpha<2 \pi$.

The accuracy of the method for the azimuthal deformations has been assessed by applying it to the streamwise station $X=0.75$ of the unforced jet interface at $\mathrm{Re}=425$. Under these conditions, azimuthal deformations are invisible to the naked eye and the jet is perfectly axisymmetric. The procedure then yields the estimate $a \sim 0.013$ for azimuthal amplitudes and this value corresponds to the minimum threshold of detection.

\section{QUANTITATIVE EVOLUTION WITH SWIRL}

The objective of the section is to document the effet of swirl on the dynamics of unforced jets in the range $0 \leqslant S$ $\leqslant 1.3$. In the entire range, the axisymmetric mode $m=0$ associated with the formation of vortex rings is prominent. The downstream evolution of the corresponding local Strouhal number $S t_{l}(X)=f_{l} D / U_{x}^{S=0}\left(0, x_{0}\right)$ is illustrated in Fig. 5 for $\operatorname{Re}=1490$ and various swirl parameter values $S$. Note that the reference velocity is taken to be the axial velocity $U_{x}^{S=0}\left(0, x_{0}\right)$ in the absence of swirl. Without swirl, random pairing events along the jet axis classically lead to a continu- 


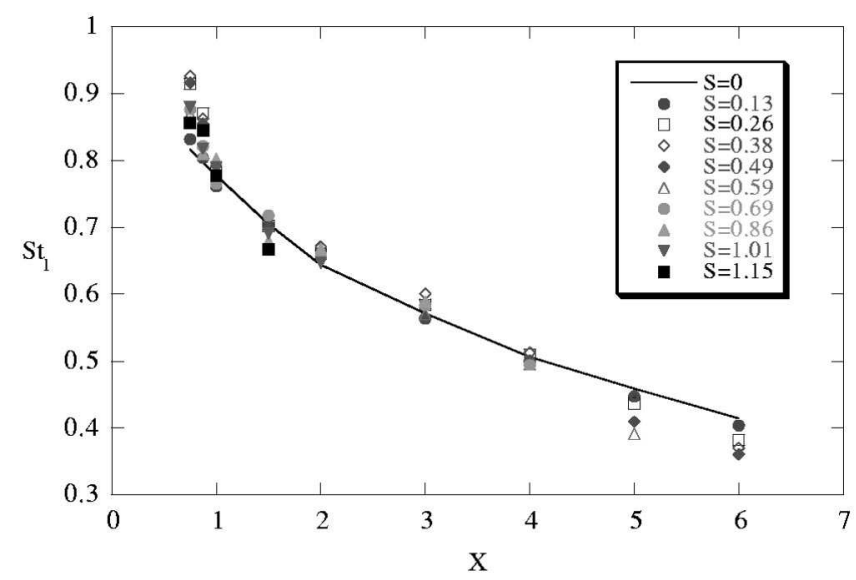

FIG. 5. $\mathrm{Re}=1490$. Spatial evolution of the local Strouhal number $S t_{l}$ for $S=0,0.13,0.26,0.38,0.49,0.59,0.69,0.86,0.94,1.01$, and 1.15 .

ous decrease in $S t_{l}$ down to the preferred jet value close to 0.4 at $X \sim 6$, at the end of the potential core (Ho and Huerre $\left.{ }^{21}\right)$. With swirl, the flow is still dominated by vortex rings and the same general trend is observed as shown in Fig. 5. Surprisingly, swirl does not seem to significantly affect the value of the frequency associated with the axisymmetric mode $m=0$

There are, however, noticeable differences that are not clearly apparent in Fig. 5. Let $L_{p}$ denote the length of the potential core scaled with respect to $D$. In the present context $L_{p}$ is operationally defined as the minimum axial distance $X$ over which spatio-temporal $r-t$ diagrams of the type illustrated in Fig. 4 first exhibit pinching of the fluorescein dyed central vertical band. Variations of $L_{p}$ with swirl $S$ are displayed in Fig. 6. For solid circles, there is no pinching, whereas, for open circles, pinching takes place. The potential core length $L_{p}$ lies somewhere in between. Two distinct regimes are clearly identifiable: when $S<0.6$, the potential core length $L_{p}$ remains approximately equal to its zero-swirl value $5.5<L_{p}<6$. When $S \geqslant 0.6, L_{p}$ gradually decreases down to the value $2.5-3$ for $S=1.26$. The pronounced de-

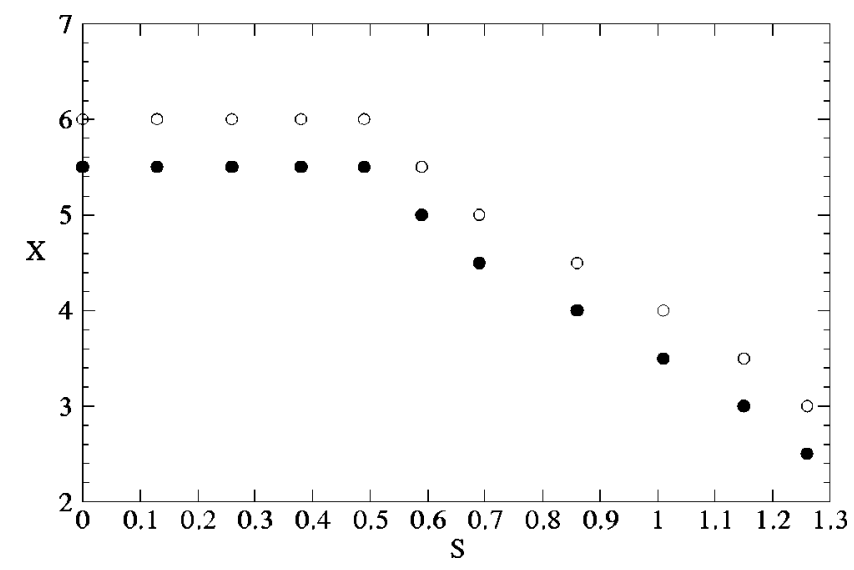

FIG. 6. Evolution of the potential core length $L_{p}$ with increasing swirl at $\operatorname{Re}=1490$. Solid circles represent the maximum axial distance $X$ where no pinching, as defined in the text, takes place. Open circles represent the minimum axial distance $X$ where pinching takes place. The potential core length $L_{p}$ lies somewhere in between.

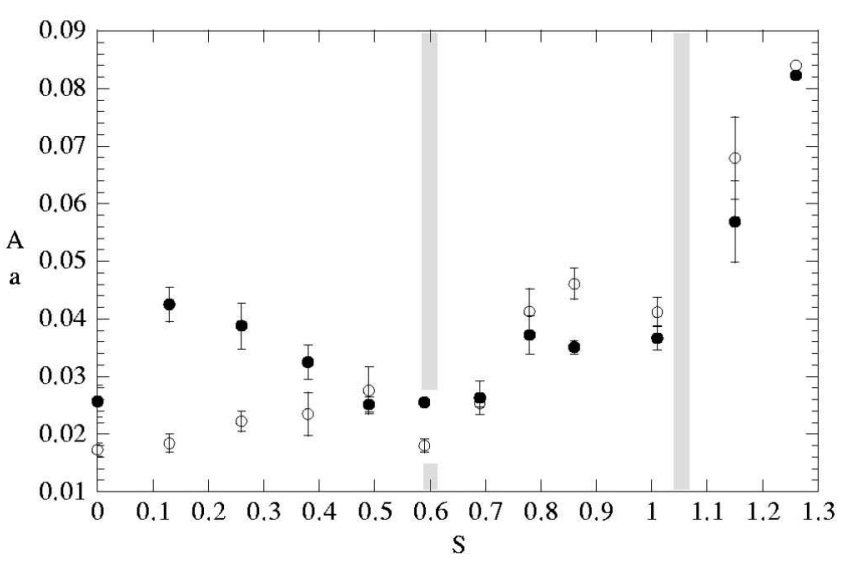

FIG. 7. Amplitudes of the axisymmetric (O) and azimuthal $(\bigcirc)$ deformations versus swirl $S$ at $\operatorname{Re}=1490$ and $X=1$.

crease of $L_{p}$ with $S$ accounts for the lack of Strouhal number data for $X>L_{p}$ at the larger swirl settings in Fig. 5.

The respective amplitude $A(X ; S)$ and $a(X ; S)$ of the axisymmetric and azimuthal deformations defined in Eqs. (7) and (8) are represented in Fig. 7 as a function of swirl $S$ at the axial station $X=1$. Three different regimes may be distinguished: in the first range $S<0.6$, axisymmetric deformations $A(1 ; S)$ are gradually lowered as swirl increases, except for very low $S$ as discussed below. The decreasing trend is in qualitative agreement with linear temporal instability analysis that predicts a decreasing growth rate of the axisymmetric mode with swirl (see Fig. 5 of Loiseleux, Chomaz, and Huerre $\left.^{13}\right)$. Note, however, the abrupt increase of $A(1 ; S)$ between $S=0$ and $S=0.13$ suggesting that vortex ring formation in jets is greatly enhanced by a minute amount of swirl. This result contradicts linear instability theory but it is consistent with the observations made by Northam at NASA Langley Research Center as reported by Khorrami: ${ }^{22}$ these authors find that the addition of low amounts of swirl to a generic combustor flowfield increases the entrainment rate of shear layers. In the same range of swirl, the amplitude $a(1 ; S)$ of the azimuthal deformations increases continuously until it reaches a value close to the amplitude $A(1 ; S)$ of the axisymmetric deformations. As $S$ increases from 0.49 to $0.59, a(1 ; S)$ decreases abruptly to a small value comparable to the amplitude $a(1 ; 0)$, and hardly larger than the detection threshold 0.013 .

In the second range $0.6<S \leqslant 1$, axisymmetric and azimuthal deformations increase significantly towards relatively high constant levels. The amplitude $a(1 ; S)$ for azimuthal deformations is larger than $A(1 ; S)$ for the axisymmetric mode.

In the last range $1<S<S_{c}=1.3$, the amplitudes $A(1 ; S)$ and $a(1 ; S)$ increase rapidly. At the highest swirl value $S$ $=1.26$ considered in Fig. 7, strong azimuthal roll-up takes place at $X=1$ (see discussion of Sec. IV D) and the measurement procedure for $a$ and $A$ described in Sec. II is no longer applicable. To offset this lack of data, the known values for $A(0.75 ; 1.25)$ and $a(0.75 ; 1.25)$ were extrapolated to the streamwise station $X=1$ by making use of the spatial growth rate prevailing at the lower swirl value $S=1.15$. 


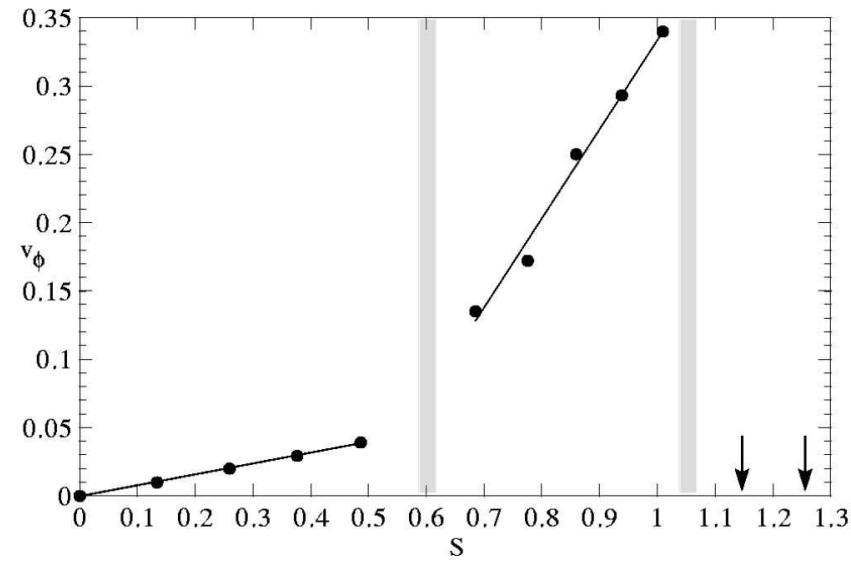

FIG. 8. Phase velocity $v_{\phi}\left(\mathrm{rad} \mathrm{s}^{-1}\right)$ of propagating helical waves $m$ versus swirl $S$ at $\operatorname{Re}=1490$ and $X=1$. The corresponding azimuthal wave number $m$ is given in Fig. 9. Vertical arrows indicate large negative values as specified in the text.

It should be emphasized that the previously measured azimuthal deformation levels are associated with identifiable travelling azimuthal waves throughout the range $0<S<1.3$. The azimuthal phase velocity $v_{\phi}$ of the dominant helical wave of highest amplitude is documented in Fig. 8 as a function of $S$. The corresponding order $m$ of the dominant azimuthal wave number is displayed in Fig. 9. Here again, three distinct regimes may be distinguished. In the absence of swirl, the jet exhibits seven stationary deformations at Re $=1490$. In the first range $0<S<0.6$, an azimuthal wave starts to propagate cyclonically as swirl increases from zero, with a phase velocity that increases proportionally with swirl. The azimuthal wave number decreases from $m=7$ for $S=0$ to $m=4$ for $S=0.49$. As $S$ reaches the value $S=0.6$, the azimuthal wave disappears. Low azimuthal deformations are still detected (Fig. 7) but they do not correspond to any identifiable propagating wave. In the second range $0.6<S$ $\leqslant 1$, the phase velocity increases at a much more rapid rate and it is no longer directly proportional to swirl. The azimuthal wave number remains constant and equal to $m=2$. In the third range $1<S<S_{c}=1.3$, a bending mode $m=1$ is observed to propagate with a high negative azimuthal phase

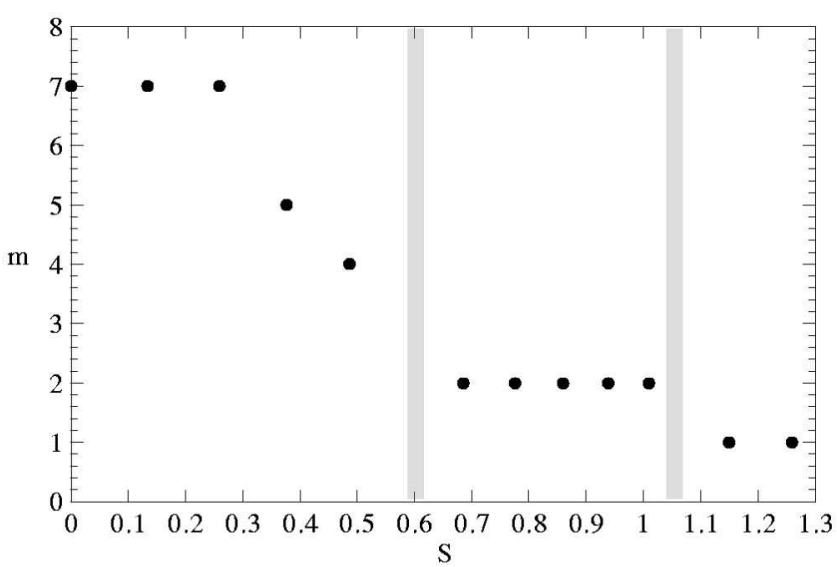

FIG. 9. Dominant azimuthal wave number $m$ versus swirl $S$ at $\operatorname{Re}=1490$ and $X=1$. velocity $v_{\phi}$. In other words, the wave travels anticyclonically with respect to the imposed rotation. For $S$ $=1.15, \quad v_{\phi}=-2.18 \mathrm{rad} \mathrm{s}^{-1}, \quad$ and for $S=1.26, \quad v_{\phi}$ $=-2.36 \mathrm{rad} \mathrm{s}^{-1}$. These values are ten times larger than in the first and second regimes and for clarity, they have only been indicated by two vertical arrows in Fig. 8 .

The azimuthal phase velocity $v_{\phi}$ is found to remain constant with downstream distance $X$ in the range $0 \leqslant X \leqslant L_{p}$. The dominant wave number $m$ exhibits only slight variations with $X$ and only in the first regime $0 \leqslant S<0.6$. For instance, for $S=0.38$, the dominant azimuthal wave number detected at $X=1$ is $m=6$, whereas at $X=4$ it is $m=5$ or 6 and at $X$ $=5$, it is $m=5$.

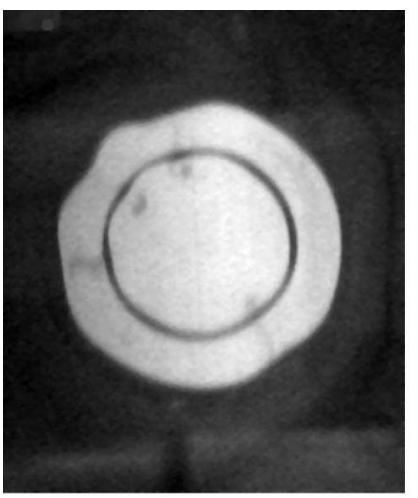

(a) ring

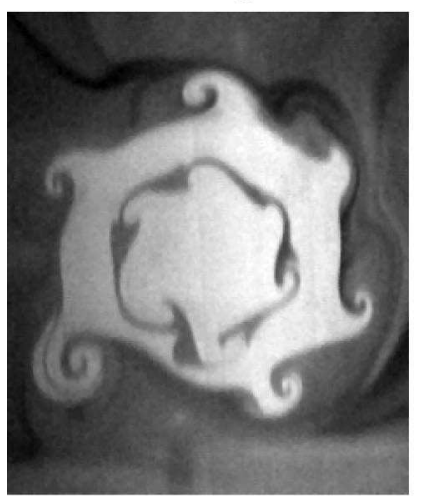

(c) ring

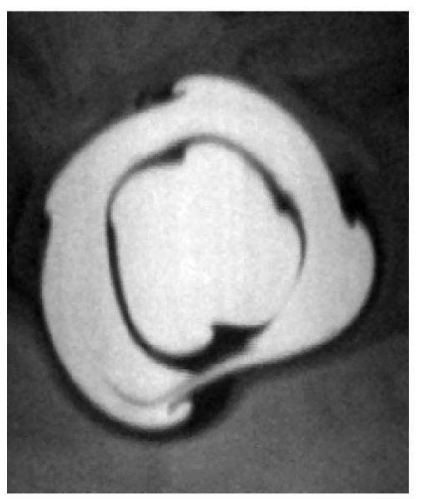

(e) ring

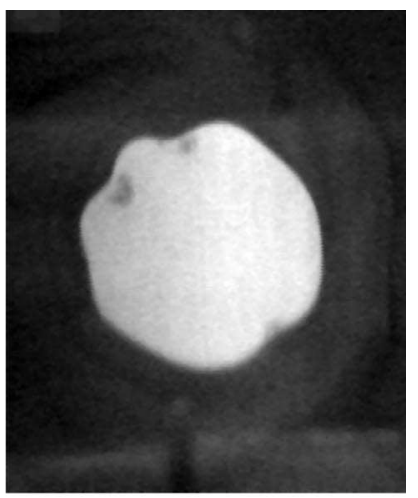

(b) braid

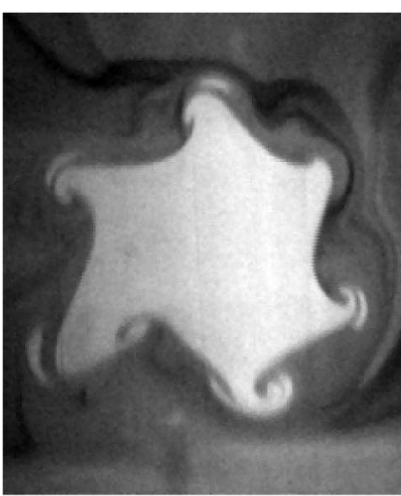

(d) braid

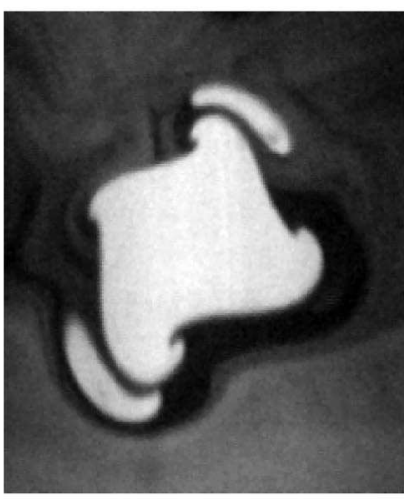

(f) braid
FIG. 10. $\operatorname{Re}=1490 . X=3$. Cross-sectional images of the jet in the ring and in the braid. (a)-(b) $S=0$, (c)-(d) $S=0.38$, (e)-(f) $S=0.49$. 


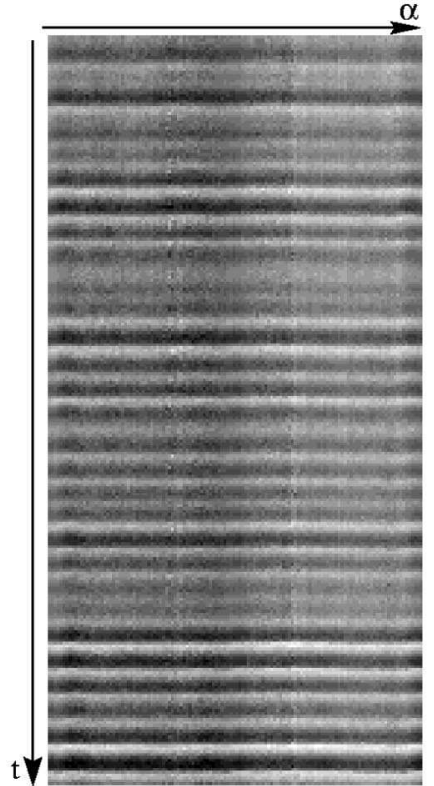

(a)

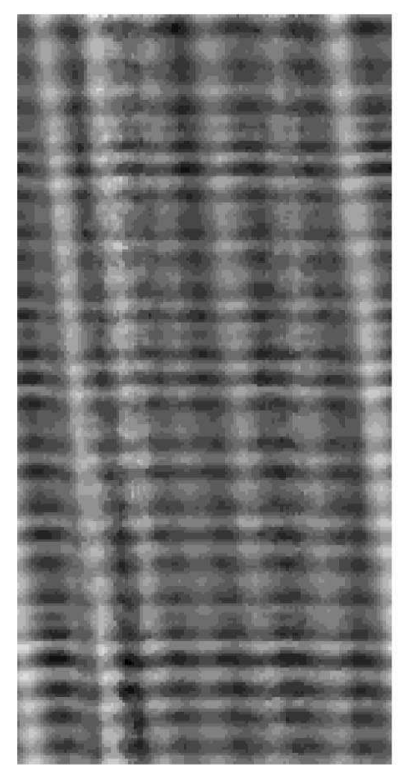

(b)

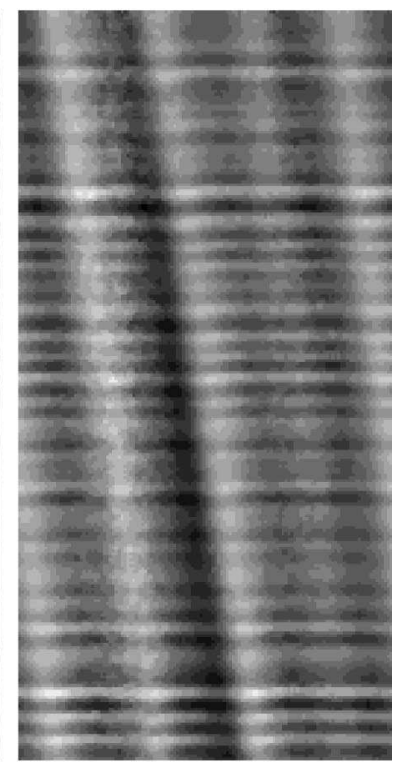

(c)
FIG. 11. $\operatorname{Re}=1490 . \quad X=1$. Spatiotemporal $\alpha-t$ diagrams for (a) $S=0$, (b) $S=0.38$, (c) $S=0.49$. The sampling rate is $\Delta t=0.16 \mathrm{~s}$. The total recording time is $T=40.8 \mathrm{~s}$.

\section{QUALITATIVE DESCRIPTION OF FLOW REGIMES}

The section is devoted to a phenomenological description of the three different flow regimes that have previously been identified.

\section{A. Low swirl regime: $S<0.6$}

The discussion relies on cross-sectional images of the jet at $X=3$ (Fig. 10) and on spatio-temporal $\alpha-t$ diagrams at $X=1$ (Fig. 11) for various swirl parameter values.

In the non swirling jet case, cross-sectional images at $X=3$ [Figs. 10(a)-10(b)] reveal the existence of axisymmetric ringlike structures and of azimuthal deformations both in the ring [Fig. 10(a)] and in the braid [Fig. 10(b)] connecting two successive rings. Rings correspond to the development of the Kelvin-Helmholtz instability associated with the shear in axial direction. The slight azimuthal deformations are generated via a secondary instability mechanism as discussed in the Introduction. Further downstream, these deformations develop into mature mushroomlike structures com-

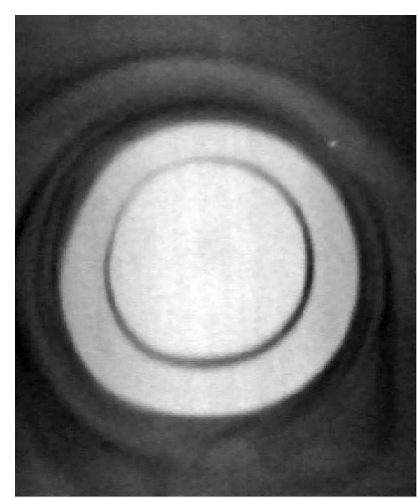

(a) ring

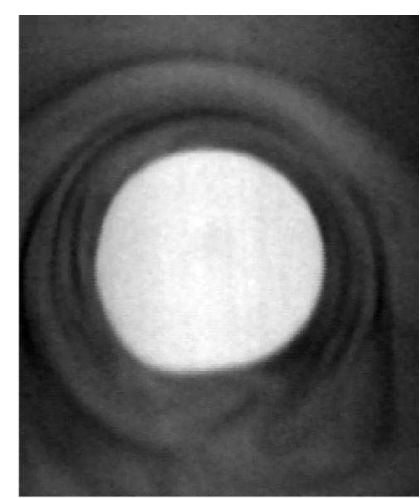

(b) braid
FIG. 12. Cross-sectional images of the jet in the ring (a) and in the braid (b) at $\operatorname{Re}=1490, X=3$, and $S=0.59$. Same scale as Fig. 10 . posed of counter-rotating streamwise vortex pairs (not shown on the figure). The corresponding $\alpha-t$ spatio-temporal diagram at $X=1$ is displayed in Fig. 11(a). The set of horizontal stripes is the imprint of the vortex ring development. The azimuthal uniformity of this diagram indicates that no azimuthal deformations are present at this distance from the nozzle exit.

In the swirling jet case, typical cross-sectional images in the ring and in the braid regions at $X=3$ are displayed in Figs. $10($ c) $-10($ d) for $S=0.38$, and Figs. 10(e)-10(f) for $S$ $=0.49$. The imposed rotation is clockwise. Corresponding $\alpha-t$ spatio-temporal diagrams at $X=1$ are documented in Figs. 11(b)-11(c). The principal quantitative results presented in Sec. III are fully confirmed and illustrated. Comparison between images at $X=3$ without swirl [Figs. 10(a)$10(\mathrm{~b})]$ and with swirl at $S=0.38$ [Figs. 10(c)-10(d)] and at $S=0.49$ [Figs. 10(e)-10(f)] indicates the following features: in the absence of swirl seven weak azimuthal deformations are barely identifiable, whereas for $S=0.38$ and $S=0.49$, respectively, six and four intense azimuthally rolled-up structures are clearly visible: swirl is observed to strengthen the development of azimuthal deformations and to cause a decrease in the azimuthal wave number $m$. The inclined stripes in the $\alpha-t$ spatio-temporal diagrams of Figs. 11(b)-11(c) for $S=0.38$ and $S=0.49$ demonstrate that a travelling azimuthal wave with a very well defined wave number $m$ and phase velocity $v_{\phi}$ is superimposed over the horizontal set of stripes already present without swirl [Fig. 11(a)]. The regularity of the phase velocity is such that $v_{\phi}$ may be determined directly by measuring the slope of the inclined stripes in the $\alpha-t$ spatio-temporal diagrams. The number of stripes at a fixed time corresponds to the order of the azimuthal mode $m$. Such an azimuthal wave corresponds to the advection by the base flow rotation of the azimuthal deformations already present in the nonrotating case.

In the first regime $S<0.6$, the underlying dominant in- 
stability mechanisms are direct extensions of those prevailing in classical nonrotating jets: the primary shear instability induces the formation of vortex rings, while a secondary instability induces azimuthal deformations that rotate proportionally with swirl, as demonstrated in Fig. 8.

However, significant differences appear in the development of the secondary instability in the presence of rotation. As discussed in Sec. III (Fig. 7), azimuthal deformations are much more intense when the jet is swirling: rotation enhances the concentration of braid vorticity into secondary streamwise structures (Fig. 10). Another striking feature is the asymmetry of the streamwise vortex pairs. Images of the swirling jet for $S=0.38$ [Figs. 10(c)-10(d)] and $S=0.49$ [Figs. 10(e)-10(f)] reveal that the azimuthal roll-up is of one sign, thereby indicating that streamwise vortices in the braids are all co-rotating one with respect to the other, and anticyclonically with respect to the base flow rotation. By contrast, in the nonswirling case, streamwise vortices are counter-rotating pairs (see for instance Liepmann and $\left(\right.$ Gharib $\left.^{23}\right)$. This result is in agreement with Martin and Meiburg's ${ }^{16}$ numerical simulations of a swirling jet subjected to axisymmetric and azimuthal perturbations. For low swirl, these authors observe the formation of vortex rings connected by co-rotating anticyclonic streamwise vortices in the braids. Similarly, when a plane mixing layer is subjected to cross-shear by suitably tilting a tank, as in Atsavapranee and Gharib's ${ }^{24}$ experiments, co-rotating streamwise vortices are observed in the braids connecting the primary spanwise rollers.

\section{B. Transitional swirl regime: $S \sim 0.6$}

When swirl reaches the value $S=0.6$, the dynamics of the swirling jet is radically different than in the previous regime. Images of the swirling jet in the ring and in the braid (Fig. 12) at $X=3$ reveal the persistence of vortex rings with only slight azimuthal deformations. This feature is confirmed by the $\alpha-t$ spatio-temporal diagram at $X=1$ displayed in Fig. 13: in contrast with Figs. 11(b)-11(c) of the preceding regime, there are no identifiable travelling azimuthal waves. This observation is also consistent with the fact that in Fig. 7,

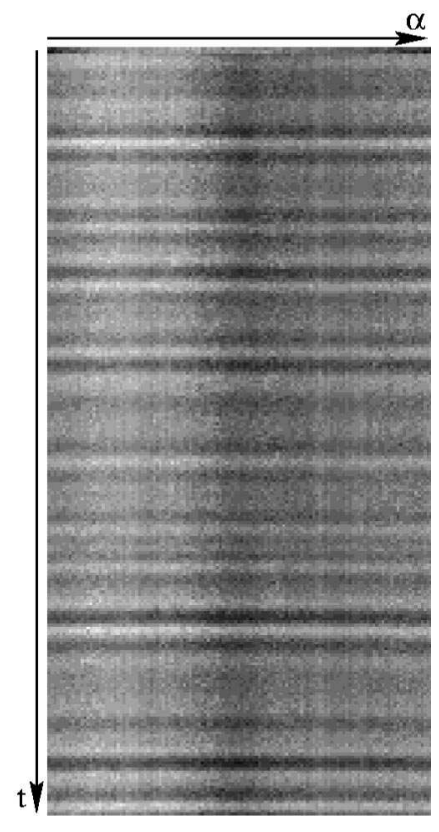

FIG. 13. Spatio-temporal $\alpha-t$ diagram at $\operatorname{Re}=1490, X=1$, and $S=0.59$. The sampling rate is $\Delta t=0.16 \mathrm{~s}$. The total recording time is $T=40.8 \mathrm{~s}$.

the azimuthal deformation amplitude at $X=1$ drops to a low value close to the level obtained without swirl, and barely above the detection threshold.

\section{Intermediate swirl regime: $0.6<S \leqslant 1$}

The quantitative results gathered in Figs. 7, 8, and 9 clearly suggest that distinct physical mechanisms are involved in the range $0.6<S \leqslant 1$. Cross-sectional images of the jet in the ring [Fig. 14(a)] and in the braid [Fig. 14(b)] reveal that the jet becomes radially elongated into a double-helix pattern as in the incipient vortex breakdown regime identified by Billant et al. ${ }^{12}$ Comparison with Figs. 10 and 12 indicates that the jet is markedly more extended in the radial direction at the same streamwise station. This implies that the jet spreading rate is effectively larger than at lower swirl levels.

In this second regime, the jet is essentially dominated by

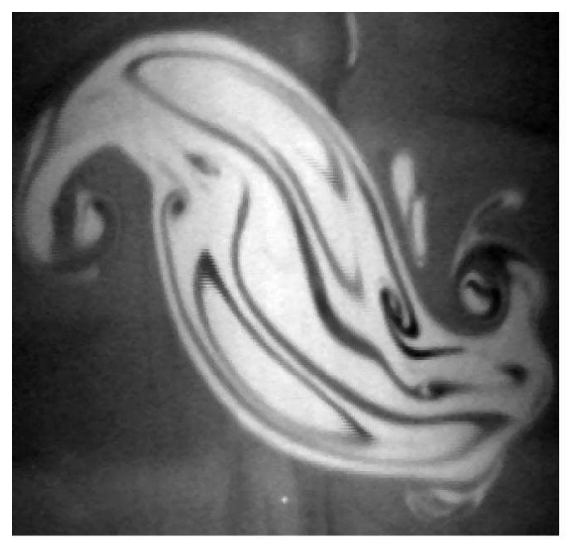

(a) ring

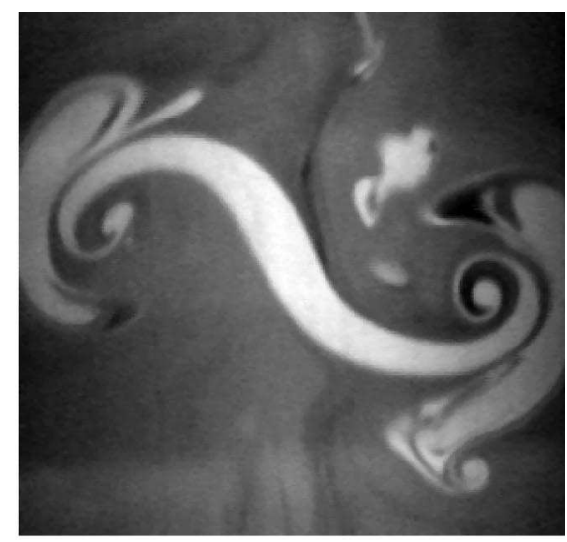

(b) braid
FIG. 14. Cross-sectional images of the jet in the ring (a) and in the braid (b) at $\operatorname{Re}=1490, X=3$, and $S=0.86$. Same scale as in Fig. 10. 


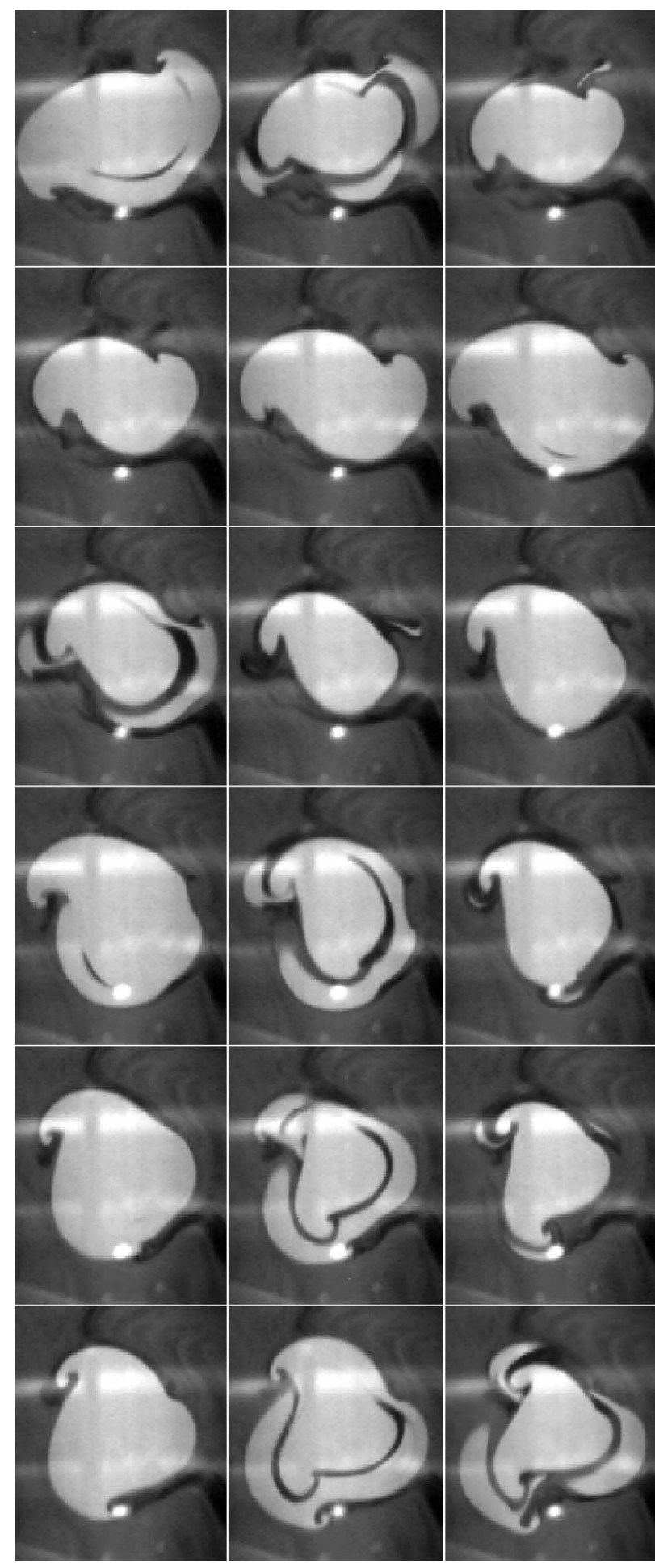

FIG. 15. Time sequence of cross-sectional images at $\operatorname{Re}=1490, X=2$, and $S=0.86$. The time step between two successive images is $\Delta t=0.48 \mathrm{~s}$.

the azimuthal mode $m=2$. However, as illustrated by the time sequence of Fig. 15 at $X=2$, a mode 3 azimuthal structure appears in a transient manner during the time evolution (see bottom frames of Fig. 15).

The $\alpha-t$ spatio-temporal diagrams at $X=1$ (Fig. 16) confirm the development of a double helical pattern but they also demonstrate that the dominant azimuthal wave does not propagate with a regular phase velocity $v_{\phi}$, as in the low

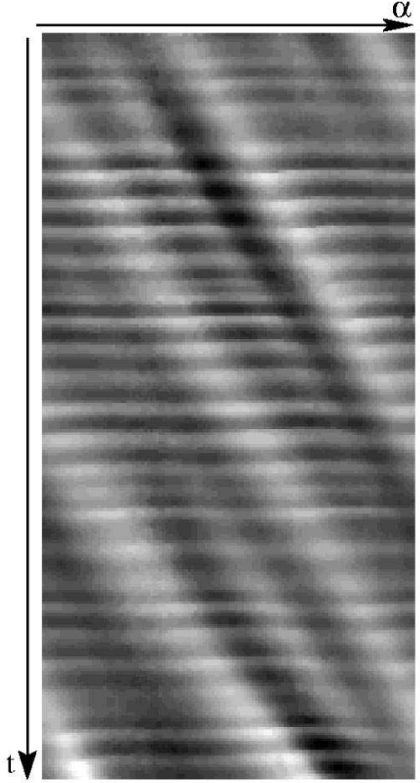

(a)

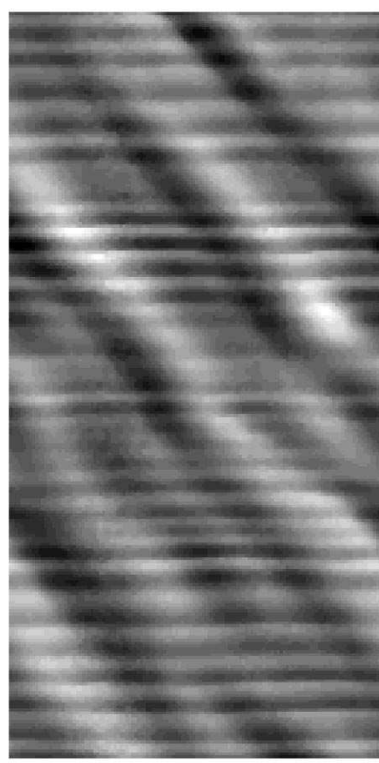

(b)
FIG. 16. $\operatorname{Re}=1490, X=1$. Spatio-temporal $\alpha-t$ diagrams for (a) $S=0.78$ and (b) $S=0.86$. The sampling rate is $\Delta t=0.16 \mathrm{~s}$. The total recording time is $T=40.8 \mathrm{~s}$.

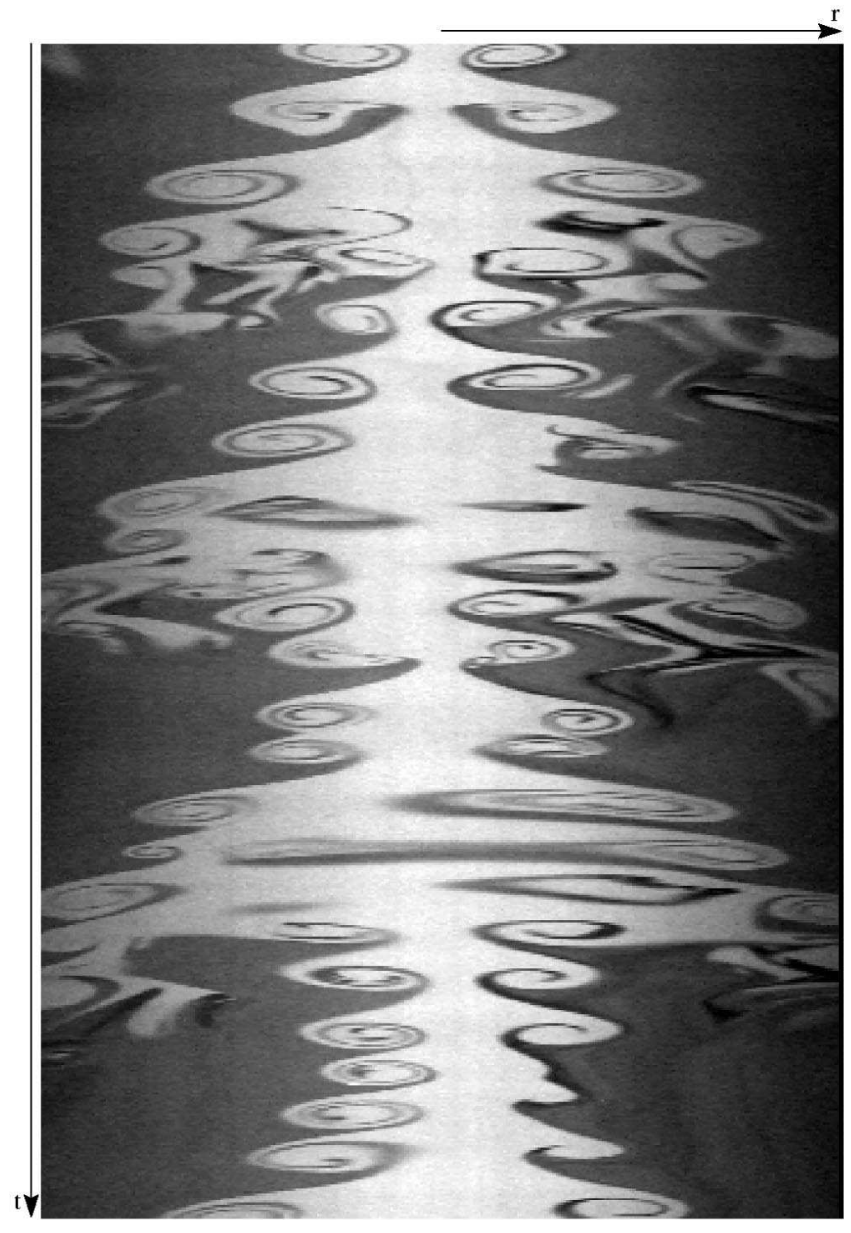

FIG. 17. Temporal evolution of jet diameter at $\operatorname{Re}=1490, S=0.86$, and $X$ $=3$. The sampling rate is $\Delta t=0.04 \mathrm{~s}$. The total recording time is $T$ $=40.8 \mathrm{~s}$. 
swirl regime of Fig. 11. The intermittent appearance of a mode $m=3$ may also be seen at the bottom of Fig. 16(b).

According to Fig. 14, the distinction between rings and braids in this swirl range may seem rather artificial and problematic. This is not really the case: as seen in the spatiotemporal $r-t$ diagram of Fig. 17, ringlike structures may still be identified and the braid region is by definition inbetween two consecutive rings. The large-scale temporal modulation evident in this figure is likely to be due to the previously identified azimuthal wave structure. The persistence of the axisymmetric mode is further confirmed by the horizontal band background present in the $\alpha-t$ diagram of Fig. 16.

A detailed examination of the flow visualization pictures in this swirl range leads to the conclusion that azimuthal roll-up in cross-sectional views tends to appear further upstream than conventional axial roll-up. For instance, at $S$ $=0.86$, azimuthal roll-up is already observed intermittently at $X=0.875$ while axial roll-up only takes place at $X$ $=1.25$. The distinction between primary and secondary instability mechanisms therefore appears to be of dubious relevance.

\section{High swirl regime: $1<S<1.3$}

In this last swirl range, strong azimuthal roll-up leads to large jet deformations close to the nozzle exit. In order to

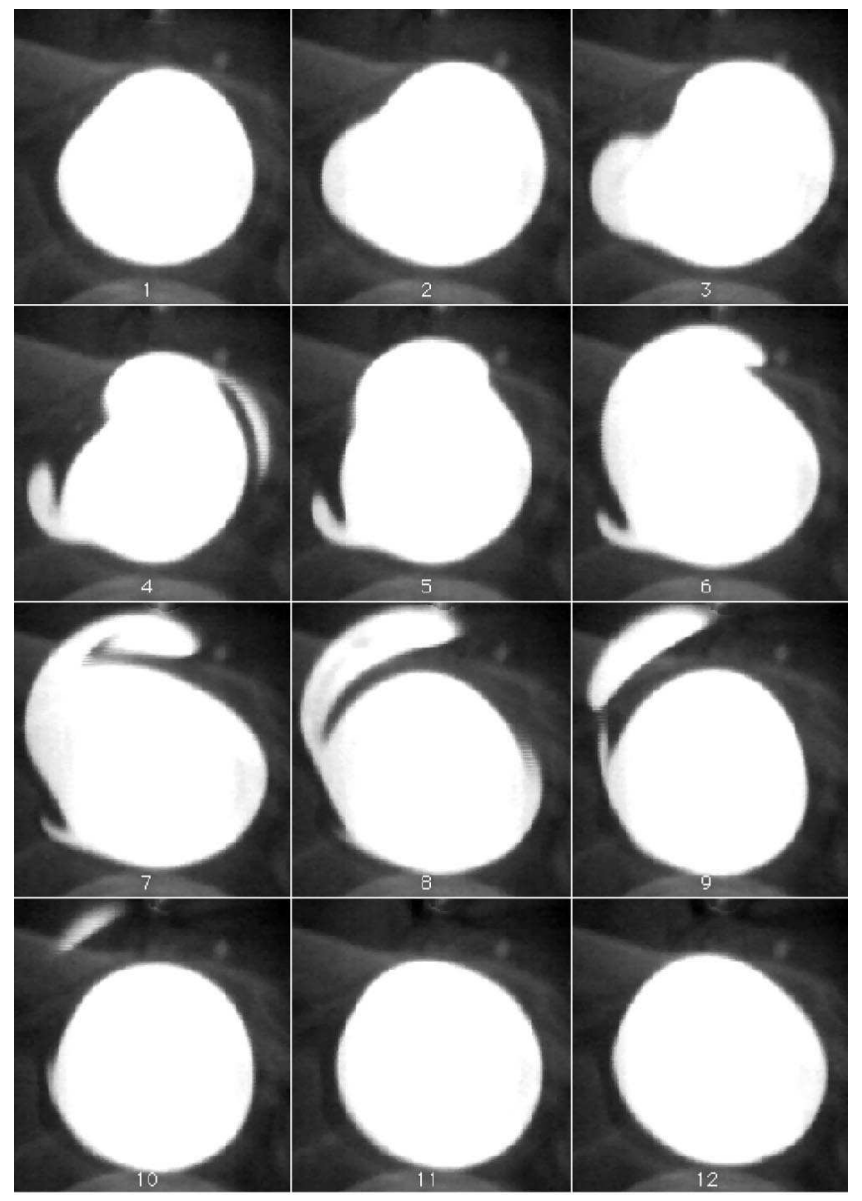

FIG. 18. Time sequence of cross-sectional images at $\mathrm{Re}=1490, X=0.875$, and $S=1.15$. The time step between two successive images is $\Delta t=0.32 \mathrm{~s}$.

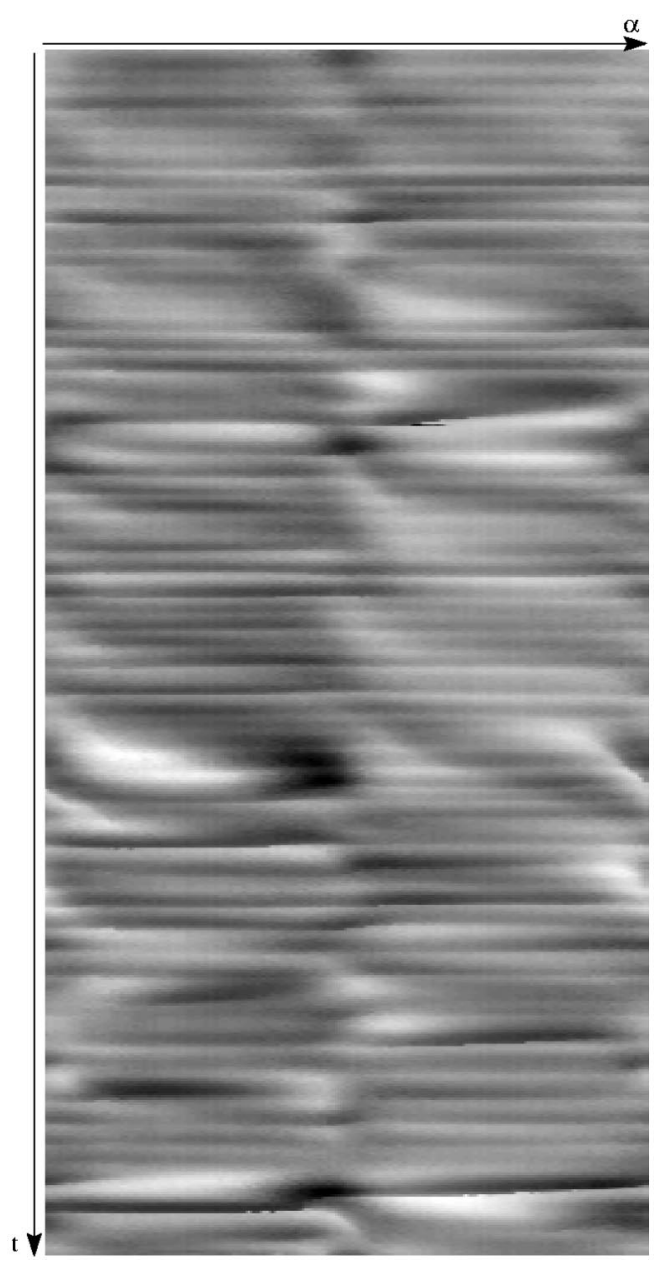

FIG. 19. Spatio-temporal $\alpha-t$ diagram at $\operatorname{Re}=1490, X=0.875$, and $S$ $=1.15$. The sampling rate is $\Delta t=0.16 \mathrm{~s}$.

extract useful qualitative informations, it is necessary to select cross-sectional views (Fig. 18) and an $\alpha-t$ diagram (Fig. 19) at streamwise stations further upstream than in the previous regimes. The temporal sequence of frames illustrated in Fig. 18 leads to a somewhat surprising observation: in contrast with the previous regimes, azimuthal deformations become cyclonic with respect to the base flow rotation and cyclonic azimuthal roll-up is already conspicuous at the downstream location $X=0.875$. Note that for lower swirl levels, azimuthal roll-up is anticyclonic. The $\alpha-t$ diagram at $X=0.875$ in Fig. 19 indicates the preservation of underlying ringlike structures, with the random generation of strong transient azimuthal inhomogeneities. Clearly identifiable traveling waves may no longer be observed.

A two-dimensional fast Fourier transform of the $\alpha-t$ plot in Fig. 19 reveals two new features readily apparent in the spectral curves of Fig. 20: the emergence of a spectral peak at a negative frequency for the helical mode $m=1$, and a broadening of the spectral curves for all azimuthal wave numbers. The appearance of the negative frequency confirms that azimuthal deformations are travelling anticyclonically as documented in Fig. 19. 


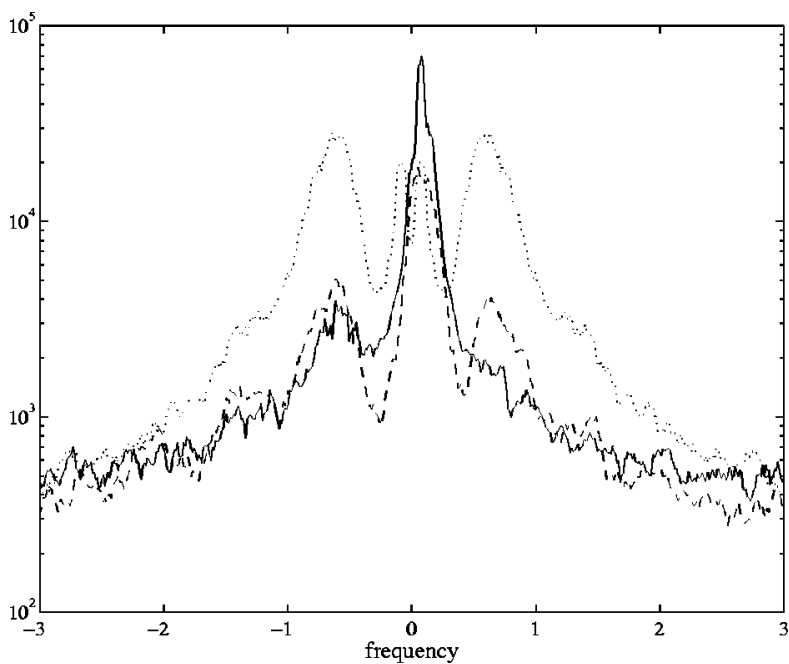

(a)

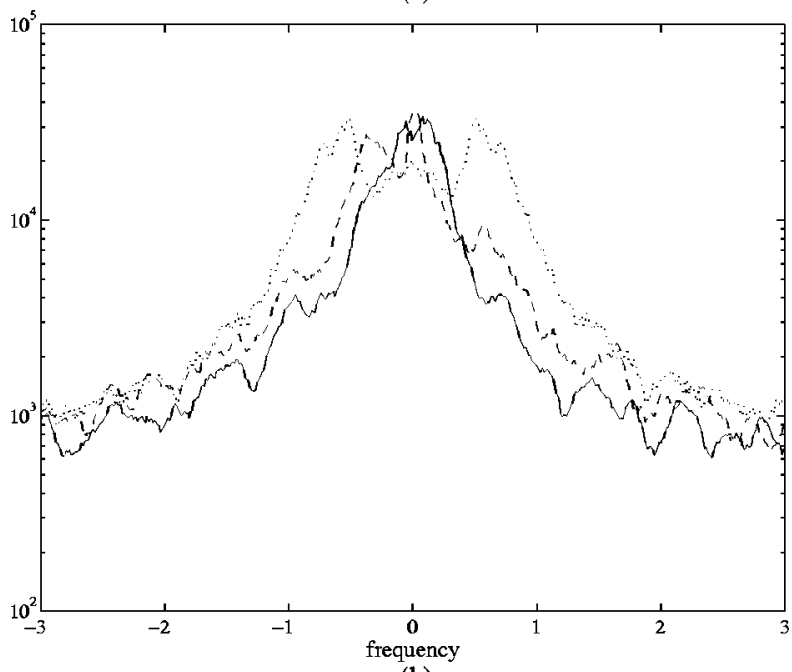

(b)

FIG. 20. Spectral curves for $m=0$ (.....), $m=1(--)$ and $m=2$ (-) at $\operatorname{Re}=1490$. (a) $S=0.86$ and $X=1$. (b) $S=1.15$ and $X=0.875$.

\section{DISCUSSION AND CONCLUDING REMARKS}

The quantitative and qualitative analyses of Secs. III and IV have led us to distinguish three dynamical regimes upstream of the potential core apex as the swirl parameter is increased in the pre-breakdown range $0 \leqslant S \leqslant 1$. . In the low swirl regime $0<S<0.6$, the axisymmetric mode $m=0$ decreases in magnitude as $S$ increases. Concurrently, a dominant helical mode of gradually decreasing azimuthal wave number (from $m=7$ to $m=4$ ) continuously grows, its azimuthal cyclonic phase velocity being proportional to swirl. Furthermore, the stationary counter-rotating streamwise vortex pairs prevailing in nonswirling jets are gradually replaced by travelling anticyclonic co-rotating streamwise vortices. This observation is consistent with the theoretical study of Lin and Corcos: ${ }^{2}$ a vortex layer with vorticity of one sign, which in the present context is imposed by the base flow rotation, generates co-rotating streamwise vortices when subjected to a strain field. The classical distinction between a primary Kelvin-Helmholtz instability leading to the formation of vortex rings and a secondary instability leading to streamwise vortices in the braids is still pertinent.
At the transitional swirl level $S \sim 0.6$, the azimuthal deformations are almost totally quenched and the flow, at least in the near field, is similar to the no-swirl case.

In the intermediate swirl range $0.6<S \leqslant 1$, the levels of the axisymmetric mode $m=0$ and helical modes $m \neq 0$ increase to reach an approximately constant plateau of comparable magnitude. Azimuthal deformations, which become larger than their axisymmetric counterparts, are composed of two traveling cyclonic waves with distinct azimuthal phase velocities with identical azimuthal wave number $m=2$. The mean phase velocity increases linearly with swirl at a much more rapid rate than in the previous regime. The shape of the jet becomes distorted into a double-helix. It appears that the primary/secondary instability distinction is no longer tenable: the shear in azimuthal direction induces azimuthal roll-up before axial roll-up is completed, and the dynamics seems to involve a competition between two primary instability mechanisms, respectively, associated with the axial and azimuthal velocity distributions.

In the high swirl regime $1<S<1.3$ immediately preceding vortex breakdown, both axisymmetric and helical modes resume their growth with increasing swirl. Although vortex rings still persist, the dominant mode is $m=1$ : it propagates anticyclonically with a large phase velocity but its appearance is sporadic and localized. According to Billant et al. ${ }^{12}$ the azimuthal velocity distribution prevailing in the rotating jet satisfies the Rayleigh criterion of decreasing circulation away from the jet axis. The flow is therefore potentially centrifugally unstable and this mechanism may be active in conjunction with the shear in axial and azimuthal direction instability mechanisms, particularly in the intermediate and high swirl ranges.

\section{ACKNOWLEDGMENTS}

Patrick Huerre is gratefully acknowledged for his careful reading and critical review of the manuscript. The authors wish to express their appreciation to Antoine Garcia for his technical assistance and his research enthusiasm.

${ }^{1}$ J. C. Neu, "The dynamics of stretched vortices," J. Fluid Mech. 143, 253 (1984).

${ }^{2}$ S. J. Lin and G. M. Corcos, "The mixing layer: deterministic models of a turbulent flow. Part 3. The effect of plane strain on the dynamics of streamwise vortices," J. Fluid Mech. 141, 139 (1984).

${ }^{3}$ Lord Rayleigh, "On the dynamics of revolving fluids," Proc. R. Soc. London, Ser. A 93, 148 (1916).

${ }^{4}$ J. L. Synge, "The stability of heterogeneous liquids," Trans. R. Soc. Can. 27, 1 (1933)

${ }^{5}$ L. R. Howard and A. S. Gupta, "On the hydrodynamic and hydromagnetic stability of swirling flows," J. Fluid Mech. 14, 463 (1962).

${ }^{6}$ S. Leibovich and K. Stewartson, "A sufficient condition for the instability of columnar vortices," J. Fluid Mech. 126, 335 (1983).

${ }^{7}$ M. Lessen, P. J. Singh, and F. Paillet, "The stability of a trailing line vortex. Part 1. Inviscid theory," J. Fluid Mech. 63, 753 (1974).

${ }^{8} \mathrm{M}$. Lessen and F. Paillet, "The stability of a trailing line vortex. Part 2. Viscous theory," J. Fluid Mech. 65, 769 (1974).

${ }^{9}$ M. R. Khorrami, "On the viscous modes of instability of a trailing line vortex," J. Fluid Mech. 255, 197 (1991).

${ }^{10} \mathrm{E}$. W. Mayer and K. G. Powell, "Viscous and inviscid instabilities of a trailing line vortex," J. Fluid Mech. 245, 91 (1992).

${ }^{11}$ J. Panda and D. K. McLaughlin, "Experiments on the instabilities of a swirling jets," Phys. Fluids 6, 263 (1994).

${ }^{12}$ P. Billant, J.-M. Chomaz, and P. Huerre, "Experimental study of vortex 
breakdown in swirling jets," J. Fluid Mech. 376, 183 (1998).

${ }^{13}$ T. Loiseleux, J.-M. Chomaz, and P. Huerre, "The effect of swirl on jets and wakes:linear instability of the Rankine vortex with axial flow," Phys. Fluids 10, 1120 (1998).

${ }^{14} \mathrm{~J}$. E. Martin and E. Meiburg, "On the stability of the swirling jet shear layer," Phys. Fluids 6, 424 (1994).

${ }^{15}$ P. I. Singh and M. S. Uberoi, "Experiments on vortex stability," Phys. Fluids 19, 1858 (1976).

${ }^{16}$ J. E. Martin and E. Meiburg, "Nonlinear axisymmetric and threedimensional vorticity dynamics in a swirling jet model," Phys. Fluids 8 , 1917 (1996).

${ }^{17}$ R. E. Caflisch, X. Li, and M. J. Shelley, "The collapse of an axisymmetric swirling vortex sheet," Nonlinearity 6, 843 (1993).

${ }^{18}$ M. N. Mikhail, "Optimum design of wind tunnel contractions," AIAA J. 17, 471 (1979)
${ }^{19}$ G. K. Batchelor, An Introduction to Fluid Dynamics (Cambridge University Press, Cambridge, 1967).

${ }^{20}$ The NIH Image software is developed at the U.S. National Institute of Health and available on the Internet at http://rsb.info.nih.gov/nih-image/.

${ }^{21}$ C. M. Ho and P. Huerre, "Perturbed free shear layers," Annu. Rev. Fluid Mech. 16, 365 (1984).

${ }^{22}$ M. R. Khorrami, "Stability of a compressible axisymmetric swirling jet," AIAA J. 30, 650 (1995).

${ }^{23} \mathrm{D}$. Liepmann and M. Gharib, "The role of streamwise vorticity in the near-field entrainment of round jets," J. Fluid Mech. 245, 643 (1992).

${ }^{24} \mathrm{P}$. Atsavapranee and M. Gharib, "A plane mixing layer with cross-shear," Phys. Fluids 6, 2880 (1994) 